\title{
Pragmatic Constraints on Semantic Presupposition
}

\author{
Yafa Al-Raheb \\ National Centre for Language Technology \\ School of Computing \\ Dublin City University, Ireland \\ yafa.alraheb@gmail.com
}

\begin{abstract}
The literature investigating the notion of presupposition in Discourse Representation Theory (DRT) has mainly been dubbed as being semantic (Simons 2003). This paper investigates the linguistic application of pragmatic-based constraints to the 'semantic' notion of presupposition in DRT. By applying pragmatic-based constraints to presuppositional phenomenon, we aim to defend DRT against the accusation that DRT's interpretation of presuppositional phenomenon is essentially 'semantic' and push this interpretation further towards the pragmatic side of the semantic/pragmatic interface. ${ }^{1}$
\end{abstract}

\section{Introduction}

Devising an appropriate theory of presupposition has been one of the main issues in semantics, pragmatics and most recently computational linguistics. Indeed, many theorists have argued extensively about the definition that captures the meaning of presupposition and whether presuppositions are a property of the utterance or of the speaker. Developments in dynamic semantics, as opposed to static semantics, resulting in DRT, have led to a framework suitable for the representation of linguistic phenomenon. Some of DRT's principal concerns are with finding the right truth conditions and interpretation for referential expressions, specifically anaphora. This is relevant for further investigation of 'pragmatic' presupposition because it has in fact been proposed by van der

\footnotetext{
${ }^{1}$ I gratefully acknowledge support from Science Foundation Ireland grant 04/IN/I527.
}

Sandt and Geurts (1991) that it is anaphora that lies at the basis of presupposition.

However, Simons (2003) has recently noted some pragmatic limitations in the present state of DRT. She refers in the following quotation to 'dynamic semantics', the field taken to include DRT.

Dynamic semantics does not attempt
to understand presupposition and pre-
suppositional constraints in terms of
the speaker's beliefs and intentions, or
to root presuppositional constraints in
terms of the broad goals of communica-
tors (Simons 2003: 27).

Indeed, Simons concludes that DRT is a theory of semantic and not pragmatic presupposition (2003). The criticism that DRT is only semantic is not wholly justified. While DRT stems from the need for appropriate semantic representation of discourse, DRT does recognize the importance of context in representing referents in discourse, which is generally taken to mark a pragmatic perspective on presupposition. Additionally, theories within DRT, such as the Binding Theory (Geurts 1999), have attempted to make DRT more pragmatic; in particular, Presupposition as Anaphora Theory's construction process of presupposition (van der Sandt and Geurts 1991). However, while this paper aims to show that DRT is not entirely devoid of pragmatics, it argues that DRT is in need of a more pragmatic treatment of presupposition, which (a) pays more attention to the beliefs and intentions of the speaker and the hearer and their relation to presupposition and (b) makes presuppositional constraints more precise. Further, other scholars have criticized DRT for being essentially truthconditional. Werth, for example, claims that DRT is essentially only about truthconditionality: 
[DRT's] goal is truth-conditionality and its models are minimal worlds inhabited by predicates and variables... it does not model human understanding: there is no place in it for participant roles, setting, background knowledge, purposes, even inferences (Werth 1999: 65).

Again, this criticism is not entirely just. To address the aforementioned pragmatic limitations of DRT, the aim of this paper is to address the problem of insightfully capturing pragmatic constraints on presuppositional phenomenon within the framework of DRT. To achieve this, a pragmaticallyconstrained definition of presupposition is attempted. This is followed by setting some pragmatic constraints on agents' conception of presuppositional phrases based on the agents' roles in conversation. An example of how these pragmatic constraints operate in DRT is then examined. Furthermore, an overall structure computationally encompassing these pragmatic constraints in DRT is described.

\section{Pragmatic-based Presupposition}

In linguistics the treatment of presupposition has generally been split between two camps, semantics and pragmatics. Karttunen (1973) maintains that the semantic perspective on presupposition sees presupposition as emanating from the sentence, and the pragmatic perspective as emanating from the speaker. Levinson argues that presupposition cannot be viewed as semantic in the narrow sense (based on formal logic and truth conditionality), but rather as dependent on context and 'the actual linguistic structure of sentences' (Levinson 1983: 167). Theories defining presupposition within pragmatics depend mainly on mutual knowledge (or common ground).

Within computational linguistics the treatment of presupposition falls mainly within dynamic semantics, within which DRT has developed. In the broadest terms presupposition, whether in linguistics or philosophy, has been viewed as a relation between sentences or propositions, where the presupposed proposition is not in focus in the presupposition sentence. DRT, and dynamic semantics in general, is precisely concerned with such relations - i.e. with the relations between an utterance and previous utterances in the discourse.

Given these varying definitions as to what constitutes presupposition, before attempting to in- troduce pragmatic constraints to presupposition in DRT, we must first explain what pragmatic presupposition, as used in this paper, constitutes. Two important notions come into question when assessing what constitutes presupposition, namely, the notion of givenness and the relationship between the beliefs (or the cognitive states) of the agents involved in a conversation.

\subsection{Presupposition and Givenness}

While presuppositions can be defined as that part of the utterance that is taken to be 'given' (Lambrecht 1994), the notion of 'given' needs clarifying. 'Given' means known information, information that the speaker regards as known to both speaker and hearer - information directly relating to the topic of the dialogue or as part of general background knowledge assumptions about the world. The presupposition in this sense is a reflection of the speaker's assumptions about the hearer's state of mind, i.e. the speaker assumes the hearer already knows this information (Lambrecht 1994). An alternate meaning of 'given' is when the speaker knows that the information the presupposition provides is, in fact, new to the hearer. In this case, the speaker introduces the new information 'as if' it were given, in order to indicate that the presupposed information is not the focus of the speaker's attention. Unlike Stalnaker's understanding of presupposition (Stalnaker 2002), this understanding of 'given' to include information new to the hearer means that the presupposition introduced is not necessarily part of what is often termed the 'common ground'.

Thus, we might think in terms of a speaker 'packaging' information as a presupposition (speaker presupposition). In this approach, the speaker has the choice of picking information she deems to be known to the hearer or information that she deems to be new to the hearer, knowing that to do so, she (the speaker) is communicating that the presupposition is not the focus of her utterance, but rather she would like the hearer to direct his attention to the new information provided by the rest of the utterance. For new, presupposed information to pass as given, the speaker must be aware that introducing an 'out of the ordinary' or 'remarkable' presupposition will cause problems. For instance, example (1) is less likely to cause problems than example (2) when the hearer knows that the speaker lives in the city centre. 
The car across the street from my house belongs to my neighbour.

The small jet across the street from my house belongs to my neighbour,

\subsection{Presupposition and Agents' Cognitive States}

To define pragmatic presupposition within DRT, presupposition should be understood to be a property of the agent. Lambrecht (1994) understands pragmatic presupposition as an interest in the assumptions speakers make about hearers. There are two types of agent presupposition, speaker presupposition and hearer presupposition. This is different from semantic presupposition, i.e. sentence presupposition. Agent presupposition differs from sentence presupposition in that the latter stems from sentence meaning, whereas the former attaches itself to the beliefs of the speaker and her intentions.

In essence, the effect of presupposition is to give insights about the speaker's beliefs as well as the speaker's beliefs about the hearer's beliefs. In this sense, the dynamic semantic notion of 'taken for granted' means that the speaker believes the presupposition to be either known information or information that is not the desired focus of attention. When a speaker introduces a presupposition in her utterance, she is not primarily concerned with the information the presupposition provides, but rather in the new information, the 'assertion' part, the utterance communicates.

Presupposition is related to the beliefs of the speaker. Speaker belief leads to presupposition, which indicates the beliefs of the speaker to the hearer. Presupposition is a reflection of the speaker's state of mind. What speakers presuppose gives an indication as to what speakers believe or accept (weakly believe) and what they believe hearers believe or accept within the context of a dialogue (cf. Al-Raheb 2005). This is stronger than what is generally conceded in the relevant literature (Geurts 1999).

While this view of pragmatic presupposition shares Stalnaker's (2002) view concerning the importance of beliefs and context to understanding linguistic phenomena, the present view of presupposition has a different understanding of the relationship between belief and presupposition. Stalnaker sees beliefs, not in terms of the speaker, but rather in terms of the vague term the "common ground' (cf. Al-Raheb 2005). '[T]o believe p to be common ground is to presuppose in Stalnaker's sense' as Simons (2003: 19) puts it. The view presented here takes the position that presuppositions reflect the speaker's beliefs, regardless of whether the beliefs are part of common ground or not.

\section{Pragmatic Constraints}

Having defined what is meant by pragmatic presupposition, we now move to discuss introducing pragmatic constraints on the phenomenon of presupposition in DRT. In order to enhance the pragmatic representation of presuppositional phenomenon in DRT, Gricean maxims need to be formulated in terms of pragmatic constraints on generating presuppositional utterances (speaker's perspective) and interpreting them (hearer's perspective). The maxims are reformulated in terms of the cognitive relationship between the speaker and the hearer, producing constraints on presupposition which are necessary for successful communication.

Following Grice's Cooperative Principle (Grice 1989), by adhering to the maxims, dialogue agents are being cooperative and not attempting to deceive or lie to one another (Grice 1989). The intention to communicate requires the speaker to assess her beliefs concerning the hearer's beliefs. This way of thinking about dialogue communication leads to the formulation of pragmatic constraints. It is proposed that these constraints broadly correspond to Grice's quantity and quality maxims. Section 4 describes an implementation of the pragmatic constraints introduced in this section.

To make her contribution informative (maxim of quantity), the speaker needs to follow the first pragmatic constraint placed on making an assertion (BCA1): to express an assertion the speaker needs to believe that the hearer does not hold the assertion, A, as a belief. In other words, the speaker believes the hearer does not hold the belief that A. This is similar to van der Sandt's (1992) informativity constraint, although his informativity constraint is not directly linked to beliefs. This pragmatic constraint is illustrated in the following example:

Speaker: Mia likes dancing. Hearer: Yeah I know.

In example (3) the hearer indicates previous knowledge of $\mathrm{A}$, which means that either the 
speaker is not following BCA1, or that the speaker was not aware that the hearer believes A. With each new utterance, the speaker must be aware of the BCA1.

The second pragmatic constraint placed on assertion is BCA2. Following from our assumptions concerning Grice's quality maxim, for a speaker to express an assertion, the speaker must herself believe or accept that assertion. That is to say, being cooperative, to express $\mathrm{A}$, the speaker must believe or accept that A.

Similarly, to introduce a presupposition, the speaker must include the presupposition in her beliefs or acceptance space (quality maxim), (BCP1). If the speaker is initiating a topic, the hearer has more grounds to conclude the speaker believes P (BCP1a). At the same time, the speaker must also be aware that when introducing $\mathrm{P}$, the speaker is communicating that the speaker believes $\mathrm{P}$. That is to say, if the speaker initiates the topic of $\mathrm{P}$, the hearer may assume that the speaker believes P. However, when it is the hearer's turn to become the speaker, and he refers to the presupposition, $\mathrm{P}$, introduced by the speaker, the speaker, who introduced $P$, may assume a weaker belief on the part of the hearer, namely that the hearer accepts P. Thus, presuppositions are built on the current context, which is built upon the union of beliefs and acceptance spaces of an agent. In other words, if the hearer refers to a presupposition employed previously by the speaker, the hearer (who now becomes the speaker) at least accepts $\mathrm{P}$ (i.e. holds $\mathrm{P}$ in his acceptance space) (BCP2).

For example,

$$
\begin{aligned}
& \text { S1: I must buy Vincent's wife a birthday } \\
& \text { present. } \\
& \text { H1: I didn't know Vincent was married. } \\
& \text { S2: Yes, he is. His wife likes chocolate. } \\
& \text { H2: She may also like flowers. } \\
& \text { S3: I'll buy her chocolates. }
\end{aligned}
$$

The speaker initiates the presupposition (in example (4) it is new information to the hearer) that Vincent has a wife. According to BCP1, the hearer may safely assume and indeed add to his beliefs about the speaker's beliefs that the speaker believes P (Vincent has a wife). However, when the hearer comes to refer to P (Vincent's wife), the speaker does not necessarily infer that the hearer believes Vincent is married, but rather that the hearer accepts P. Introducing a topic for the presupposition allows the hearer to add more strength to her representation of the speaker's beliefs, i.e. to establish belief rather than acceptance. This would be more evident in a context where the speaker is attempting to persuade the hearer to do something. For information dialogues, where the information provider has the authority of possessing the answers to the information seeker's questions, the beliefs of the information provider may attain a stronger position than they would in other types of social contexts (cf. Al-Raheb 2005). The information seeker is less likely to challenge or evaluate the strength of belief of the information provider. If we contrast example (4) with example (5), the strength of beliefs are much higher, we can assume, than for when the hearer is not required to perform any action.
S1: You should buy Vincent's wife a birthday present.
H1: I didn't know Vincent was married.
S2: Yes he is. His wife likes chocolate.
$\mathrm{H} 2$ : She may also like flowers.
S3: But she prefers chocolate.
H3: I'll get her some chocolate.

In example (4), where the hearer was not required to perform any action, it is safer for the speaker to assume that the hearer accepts $\mathrm{P}$ as the hearer is not committing himself to doing any task, than to assume the stronger case, i.e. the hearer believes P. However, in example (5) where the hearer agrees to buying Vincent's wife a present in $\mathrm{H} 3$ (that is the hearer commits to perform an action for Vincent's wife), the speaker will conclude that the hearer believes the presupposed proposition and adds this to the speaker's representation of the hearer's beliefs. In other words, when the hearer makes $\mathrm{P}$, in $\mathrm{H} 3$, the speaker concludes that the hearer believes $\mathrm{P}$ and adds this to the speaker's representation, or beliefs set, of the hearer's beliefs. Thus, someone getting someone else's commitment to do something implies greater strength of belief about a presupposition which affects that commitment.

Allowing the hearer to assume weaker belief brings us back to Simons' (2003) suggestion of modifying Stalnaker's understanding of presupposition to become what she terms the 'disposition of presupposition': speakers 'act as if' they take the presupposition for granted. Simons (2003) argues that speakers do not need to believe the presuppositions they use. With acceptance, as understood by the view of pragmatic presupposition 
presented in this paper, speakers do not have to hold the strong belief P. But, at the same time, to express $\mathrm{P}$, speakers should not hold the belief that $\mathrm{P}$ is false. That is, being cooperative necessitates that when the speaker utters $\mathrm{P}$, the speaker does not hold the belief that $\neg$ P. Speakers may indicate stronger belief. However, if there is no such indication (e.g. 'Definitely', or 'I couldn't agree more'), hearers may conclude that speakers at least hold the presupposition in their acceptance space. Speakers may later allow the hearer to conclude that they hold greater strength of belief than at first assumed.

\section{Implementing Pragmatic Constraints}

The following set of operations implement the pragmatic constraints on presupposition and assertion set out in section 3, namely BCA1, BCA2, $\mathrm{BCP} 1$, and $\mathrm{BCP} 2 .^{2}$ We begin, firstly, by showing how the pragmatic constraint operations are implemented and by demonstrating how the code works on linear DRSs (cf. Al-Raheb 2005). Secondly, we demonstrate how the constraints work on a real example by passing an example dialogue through the implemented pragmatic constraints for both the speaker and the hearer.

\subsection{Implementation}

The first pragmatic constraint on assertion (BCA1) is represented by beliefConstraintA1. It checks that a condition is not in the hearer's Belief DRS nor inside the hearer's Acceptance DRS in a Speaker's Belief or Acceptance DRS. Agent's Belief DRSs represent stronger beliefs they hold and stronger beliefs they have about other agents in dialogue, whereas Acceptance DRSs represent their weaker beliefs about the dialogue and about the weaker beliefs held by other agents in dialogue (cf. Al-Raheb 2005). When a condition is not found in either embedded DRS, it succeeds.

The second pragmatic constraint on assertion (BCA2) is represented by beliefConstraintA2, which checks if a condition is either in the speaker's Belief or Acceptance DRS. The operation succeeds once a match is found. ${ }^{3}$ BCP1

\footnotetext{
${ }^{2}$ The implementation outlined in this section demonstrates how pragmatic constraints on presupposition can be implemented, but does not describe the entire architecture of checking that these constraints are adhered to in processing an entire dialogue.

${ }^{3}$ These pragmatic constraints on assertion are not demonstrated as the focus of this paper is presupposition.
}

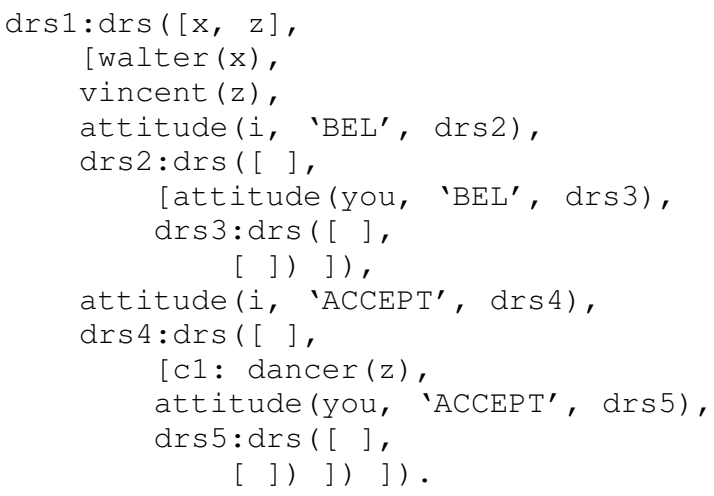

becomes:

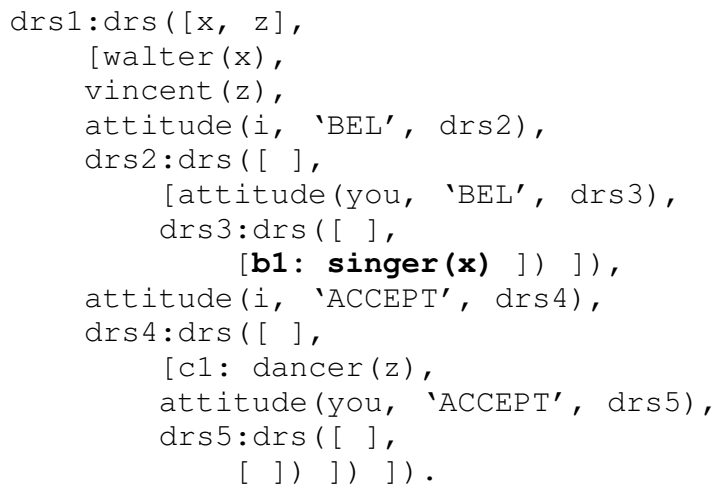

Figure 1: Hearer BCP1

has been subdivided into speaker and hearer. The speaker side, beliefConstraintSpeakerP1 checks if a condition is a member of the speaker's belief or acceptance DRS.

For the hearer, beliefConstraintHearerP1 checks if a condition is not a member of the speaker's acceptance or belief DRS, then checks if the condition is not a member of the hearer's belief or acceptance DRS. If this check passes, the condition is added to the hearer's belief about the speaker's belief, i.e. speaker's belief DRS embedded inside the hearer's DRS. When using 'beliefConstraintHearerP1' to check for the condition 'singer(X)', we get the second DRS as a result in Figure 1.

The second version of the hearer's $\mathrm{BCP} 1$, beliefConstraintHearerP1a, checks if a condition is not a member of the speaker's belief or acceptance DRS, and is a member of the hearer's belief or acceptance DRS. Then, it adds the condition to the speaker's acceptance DRS, embedded inside the hearer's acceptance DRS. This is shown in Figure 2.

The second pragmatic constraint on Presuppo- 


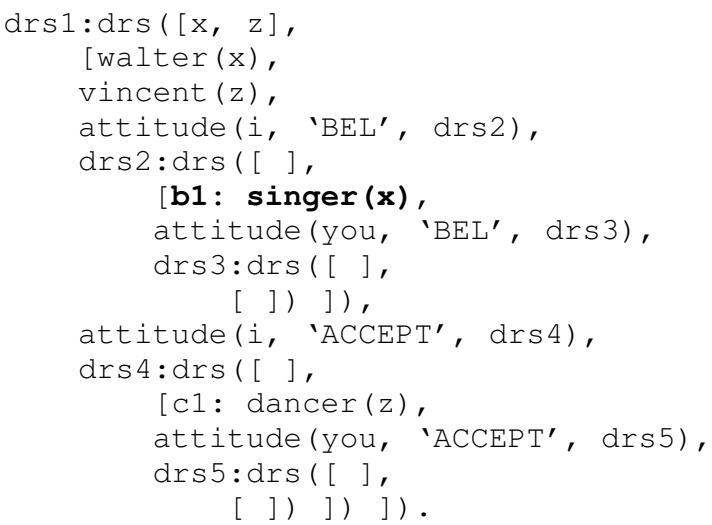

becomes:

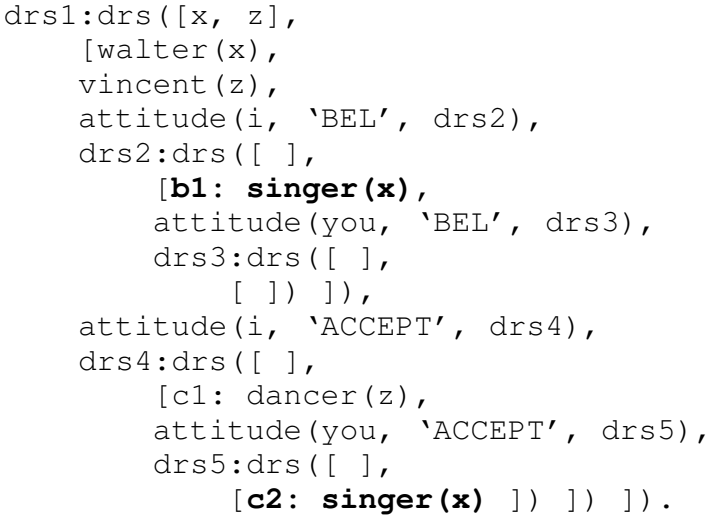

Figure 2: Hearer BCP1a

sition (BCP2) is also divided into speaker side and hearer side. The speaker's side, beliefConstraintSpeakerP2, checks a condition, e.g. 'singer $(\mathrm{X})$ ', is a member of the speaker's belief or acceptance DRS, but not a member of the hearer's acceptance or belief DRS, embedded inside the speaker's DRSs (Figure 3). Once this is fulfilled, the condition is added to the hearer's acceptance DRS, embedded inside the speaker's acceptance DRS.

$\mathrm{BCP} 2$, from the hearer's perspective, uses beliefConstraintHearerP2, which checks whether a condition, e.g. 'singer(X)', is a member of the speaker's belief or acceptance DRS (embedded inside the hearer's DRSs), and then checks if the condition is not a member of the hearer's acceptance or belief DRSs. It then adds the condition to the hearer's acceptance DRS, as in Figure 4.

\subsection{Application}

The following example

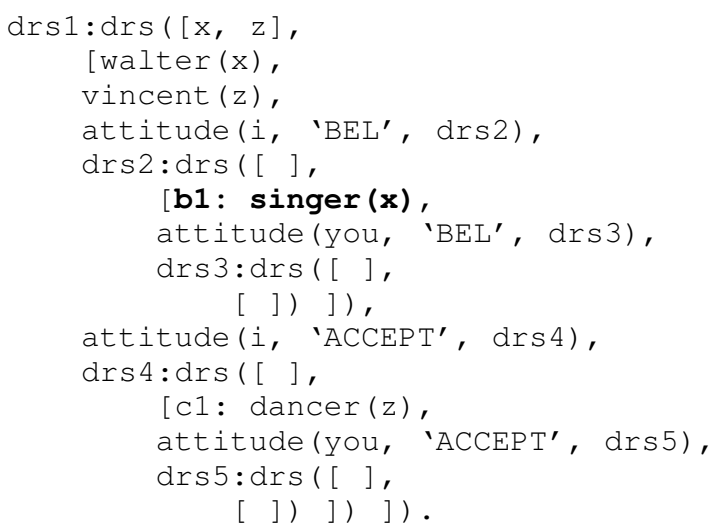

becomes:

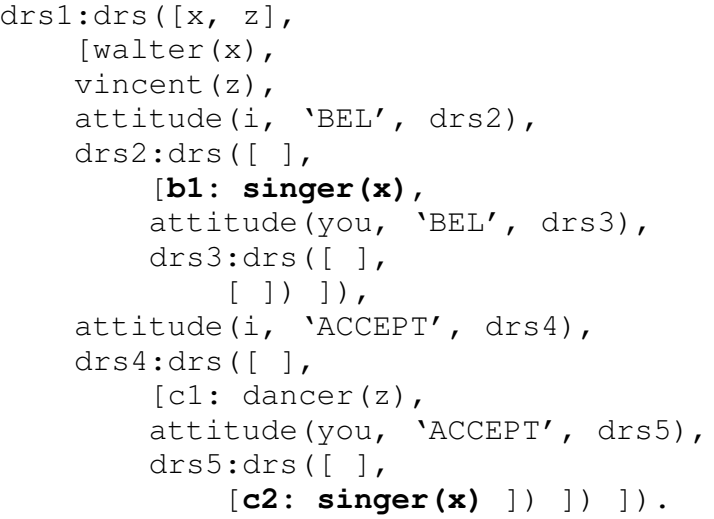

Figure 3: Speaker BCP2

(6)

S1: You have to buy Vincent's wife a present.

H1: Should I send Mia flowers?

S1: Mia likes chocolate. Buy her some.

H2: I'll buy her chocolate.

is passed through the implemented pragmatic constraints to briefly demonstrate how the pragmatic constraints can be employed in dialogue. Figures 5 and 6 show the initial beliefs of both agents before the dialogue is initiated. To proceed, the pragmatic constraints for the speaker are applied. If the pragmatic constraints do not apply, the dialogue cannot go forward. This starts a recognition process on the part of the hearer.

To utter $\mathrm{S} 1$, the speaker is constrained by $\mathrm{BCP} 1$ for the speaker, which dictates that the presupposition to be uttered needs to be part of the speaker's beliefs or acceptance DRSs (cf. section 3). When this is verified, the speaker is able to make an utterance containing the presupposition resulting 


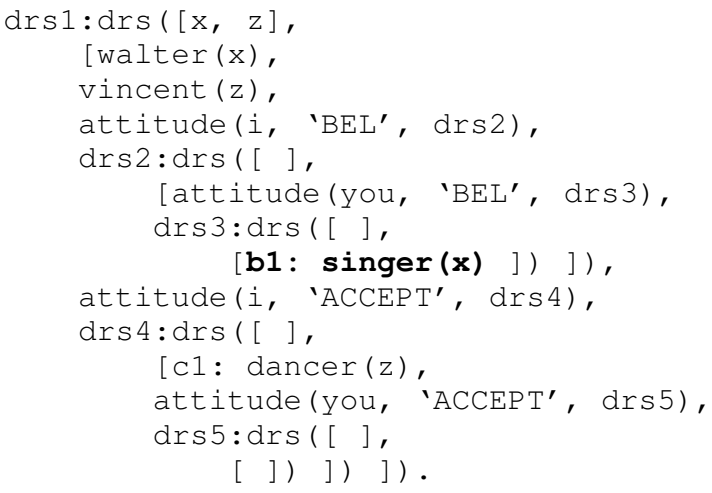

becomes:

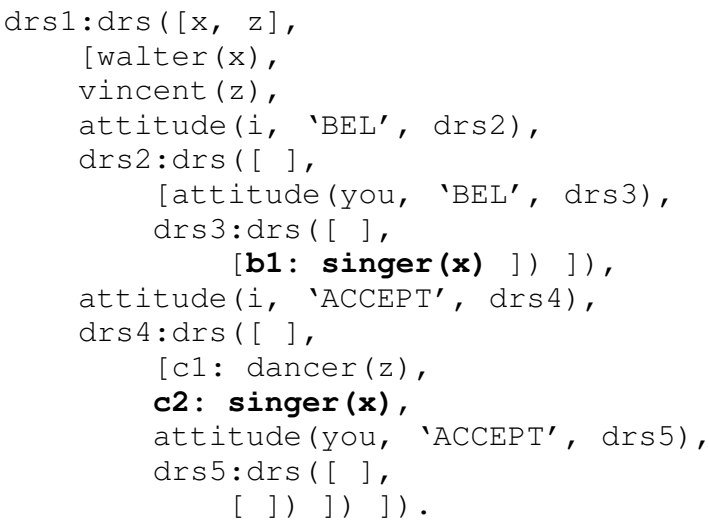

Figure 4: Hearer BCP2

in Figure $7 .^{4}$ Here, the speaker already believes the hearer believes the contents of the presupposition, 'Vincent has a wife', and as such nothing needs to be changed in the speaker's beliefs about the hearer's beliefs, BCP2 for the speaker. If, however, the speaker did not already believe the hearer believes the presupposition, the contents of the presupposition would be added to the hearer's acceptance DRS inside the speaker's acceptance DRS according to BCP2 for the speaker.

In the process of recognizing the speaker's utterance, the hearer's pragmatic constraints are employed and if they are violated, again the dialogue cannot proceed. The first pragmatic constraint for the hearer to apply is BCP1, which checks whether both the speaker and the hearer do not believe or accept the presupposition. Upon finding that both the speaker and the hearer believe the presupposition, there is no need to add anything to either the hearer's or the speaker's belief and acceptance spaces. If, however, that was not the case, BCP1 and BCP1a alter the hearer's and speaker's

\footnotetext{
tered.

${ }^{4}$ Figures 7 and 8 show both agents' beliefs after S1 is ut-
}

belief states accordingly by adding the contents of the presupposition as indicated in section 4.1. The same reasoning applies to checking whether BCP2 applies, for if the hearer did not already believe or accept the presupposition, BCP2 adds the contents of the presupposition to the hearer's acceptance DRS. After this, the speaker's pragmatic constraints are checked against the hearer's, who is now the speaker, utterance and so on.

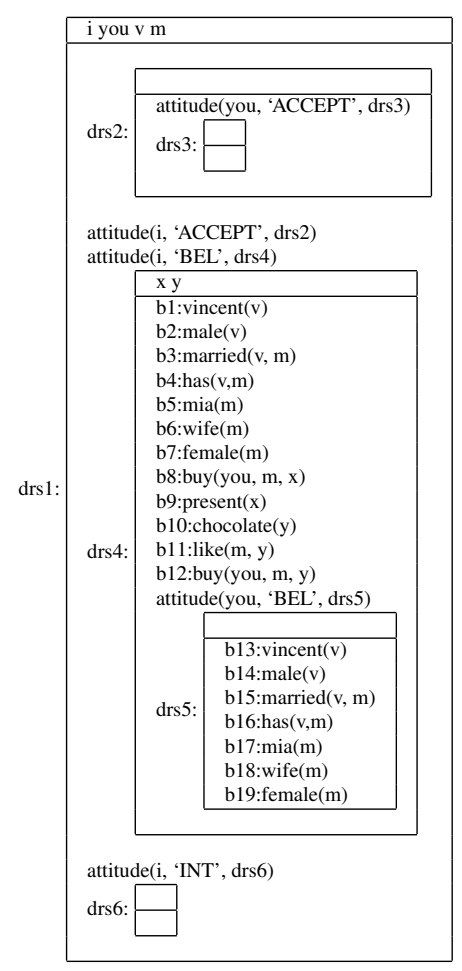

Figure 5: Speaker Initial State

\section{Conclusion}

This paper has introduced a pragmatic view of presupposition both in terms of givenness and the effects agents' cognitive states have on formulating presupposition. To introduce this pragmatic view of presupposition into DRT, some pragmatic constraints have been formulated and demonstrated by way of example. In addition, an implementation of these pragmatic constraints on presupposition has been introduced into the extended DRT representation formulated by Al-Raheb (2005).

\section{References}

Al-Raheb, Y. 2005. Speaker/Hearer Representation in a Discourse Representation Theory Model of Presupposition: A Computational-Linguistic Approach. Phd. University of East Anglia. 


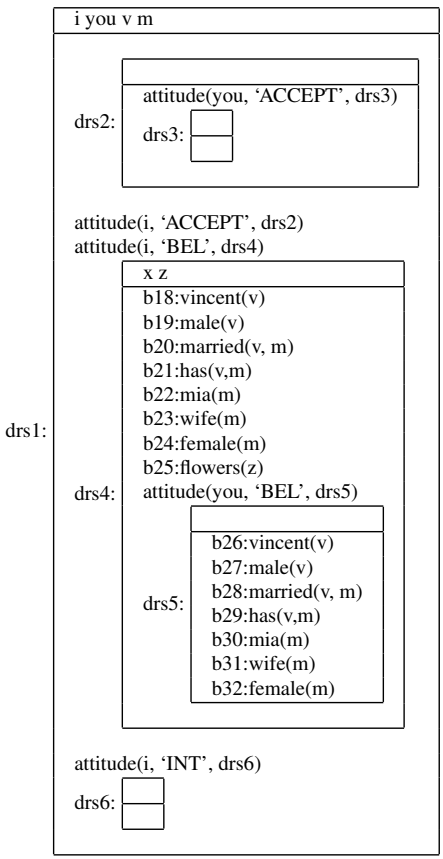

Figure 6: Hearer Initial State



Figure 7: Speaker Generation: After S1

Geurts, B. 1999. Presuppositions and Pronouns: Current Research in the Semantics/ Pragmatics Interface. Oxford: Elsevier.

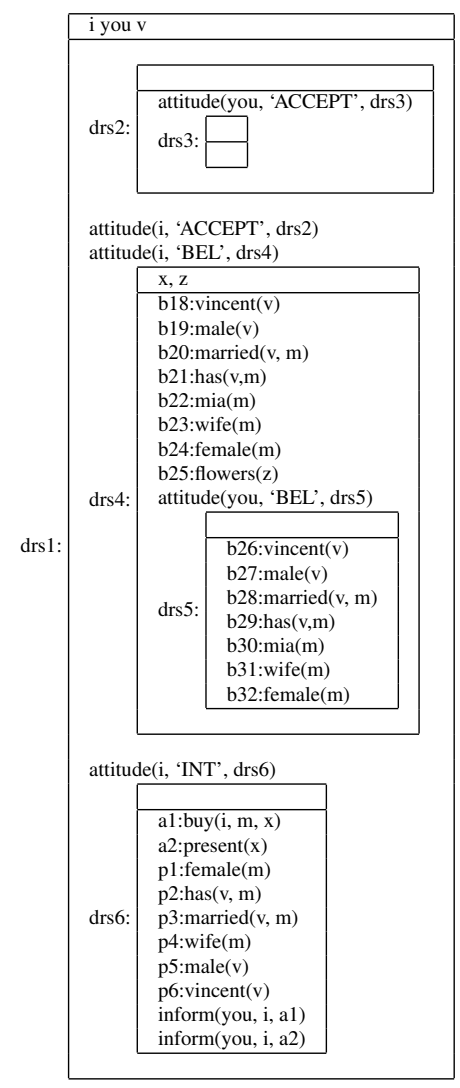

Figure 8: Hearer Recognition: After S1

Grice, P. 1989. Studies in the Way of Words. Cambridge, MA: Harvard University Press.

Lambrecht, K. 1994. Information Structure and Sentence Form: Topic, Focus and the Mental Representations of Discourse Referents. Cambridge: Cambridge University Press.

Levinson, S. 1983. Pragmatics. Cambridge: Cambridge University Press.

Simons, M. 2003. 'Presupposition and Accommodation: Understanding the Stalnakerian Picture'. Philosophical Studies 112, pp. 251-278.

Stalnaker, R. 2002. 'Common ground'. Linguistics and Philosophy 25(5-6), pp. 701-721.

van der Sandt, R. and Geurts, B. 1991. 'Presupposition, Anaphora, and Lexical Content'. In: O. Herzog and C.-R. Rollinger (Eds.). Text Understanding in LILOG. pp. 259-296. Berlin, Heidelberg: Springer Verlag.

van der Sandt, R. 1992. 'Presupposition Projection as Anaphora Resolution'. Journal of Semantics 9, pp. 333-377.

Werth, P. 1999. Text Worlds: Representing Conceptual Space in Discourse. New York: Longman. 